\title{
Stabilization of Nonholonomic Chained Systems via Nonregular Feedback Linearization
}

\author{
Z. Sun ${ }^{\dagger \ddagger}, \quad$ S. S. Ge,$^{\dagger *}$ W. Huo ${ }^{\ddagger}$ and T. H. Lee ${ }^{\dagger}$ \\ $\dagger$ Department of Electrical Engineering \\ National University of Singapore, Singapore 117576 \\ $\ddagger$ The Seventh Research Division \\ Beijing University of Aero. \& Astro., Beijing 100083, China \\ * To whom all correspondence should be sent. \\ Tel. (+65) 8746821; Fax. (+65) 7791103; E-mail: elegesz@nus.edu.sg
}

\begin{abstract}
This paper addresses the problem of feedback stabilization of nonholonomic chained systems within the framework of nonregular feedback linearization. Firstly, the nonsmooth version of nonregular feedback linearization is formulated, and a criterion for nonregular feedback linearization is provided. Then, it is proved that a chained form is linearizable via nonregular feedback control, thus enable us to design feedback control laws using standard techniques for linear systems. The obtained discontinuous control laws guarantee convergence of the closed-loop system with exponential rates. Finally, simulation results are presented to show the effectiveness of the approach.

Keywords: Nonholonomic systems; Stabilization; Nonregular feedback linearization; Discontinuous control
\end{abstract}

\section{Introduction}

Control of nonholonomic systems is theoretically challenging and practically interesting. Due to Brockett's Theorem [4], these systems cannot be stabilized to a point with pure smooth (or even continuous) state feedback control. As a consequence, the classical smooth theory and design mechanism of nonlinear control systems cannot be applied. Nonholonomic systems cover a wide range of practice systems. Nonholonomic constraints are naturally imposed on the motion of a large class of mechanical systems, typical examples including mobile robots, and cars with/without trailers $[6,10]$.

During last decade, many papers have been published on the control of nonholonomic 
systems. Many control strategies and algorithms were developed, based on either

physical (kinematic/dynamic) models [11] or canonical (chained or power) forms $[1,6,12]$. For point stabilization of nonholonomic systems, it has been shown that a time-periodic smooth feedback control cannot be exponentially stabilizing [7], while discontinuous state control or nonsmooth time-varying controllers are able to provide exponential (or finite-time) stability, see, $[3,6,8,1,2,12]$ to list a few.

In this paper, we address the problem of stabilization for nonholonomic single chained systems in the framework of nonregular feedback linearization. In Section 2, the formulation of nonregular feedback linearization [13] is generalized to a nonsmooth case to include discontinuous or singular state and feedback transformations. In Section 3, the chained systems are proved to be nonregular feedback linearizable and discontinuous controllers are designed for a wheeled mobile robot. The design mechanism is generalized to the general single chained systems in Section 4. In Section 5, simulation study is carried out to illustrate the effectiveness of the proposed control strategies.

\section{Nonsmooth Nonregular Feedback Linearization}

In this section, we present a generalized formulation of nonregular static state feedback linearization [13].

Consider the multi-input affine nonlinear systems given by

$$
\dot{x}=f(x)+\sum_{i=1}^{m} u_{i} g_{i}(x)=f(x)+G(x) u
$$

where $x \in \Re^{n}$ and $u \in \Re^{m}$. The entries of $f(x)$ and $G(x)$ are analytic functions of $x$, and $\operatorname{rank} G(x)=m, \forall x \in \Re^{n}$.

Since Krener [9], the problem of exact linearization has been studied using increasingly more general transformations. In [13], the use of nonregular state feedbacks was investigated to achieve linearization of affine nonlinear systems which is of independent theoretical interest. In this paper, a nonsmooth version of the problem of nonregular feedback linearization is introduced to cope with driftless systems. 
Definition 1 Any feedback transformation of the form

$$
u(t)=\alpha(x)+\beta(x) v(t)
$$

where $\alpha(x): \Re^{n} \rightarrow \Re^{m}, \beta(x): \Re^{n} \rightarrow \Re^{m \times m_{0}}, v \in \Re^{m_{0}}, m_{0} \leq m$, is called a nonregular static state feedback transformation.

Definition 2 Nonlinear control system (1) is said to be nonregular (static state) feedback linearizable if there exist a discontinuous state transformation

$$
z=T(x), z \in \Re^{n}
$$

and a nonregular state feedback (2), such that the transformed system with state z and input $v$ reads as a controllable linear system.

For nonregular feedback linearizability of two-input driftless systems, the results are given in Lemma 1.

Lemma 1 For a two-input driftless system

$$
\dot{x}=g_{1}(x) u_{1}+g_{2}(x) u_{2}
$$

if the nested distributions defined by

$$
\begin{aligned}
& \Delta_{0}=\operatorname{span}\left\{g_{2}\right\} \\
& \Delta_{i}=\Delta_{i-1}+a d_{g_{1}} \Delta_{i-1}, i=1, \cdots, n-2 \\
& \Delta_{n-1}=\Delta_{n-2}+\operatorname{span}\left\{g_{1}\right\}
\end{aligned}
$$

satisfy

(i) $\Delta_{i}$ is involutive and of constant rank for $0 \leq i \leq n-1$; and

(ii) $\operatorname{rank} \Delta_{n-1}=n$

then system (3) is nonregular feedback linearizable.

Proof. By Frobenius Theorem, there exist real-valued functions $\phi(x)$ and $h(x)$ : $\Re^{n} \rightarrow \Re$, such that

$$
d \phi \perp \Delta_{n-3}, d \phi \not \perp \Delta_{n-2}, d h \perp \Delta_{n-2}, \quad \text { and } L_{g_{1}} h=1
$$


Let

$$
u_{1}=\phi(x), \quad f(x)=\phi(x) g_{1}(x)
$$

System (3) can be rewritten as

$$
\dot{x}=f(x)+g_{2}(x) u_{2}
$$

Compute the following quantities

$$
\begin{aligned}
L_{g_{2}} h & =0, \quad L_{g_{2}} L_{f} h=L_{g_{2}} \phi=0 \\
L_{g_{2}} L_{f}^{2} h & =L_{g_{2}}\left(\phi L_{g_{1}} \phi\right)=\left(L_{g_{2}} \phi\right)\left(L_{g_{1}} \phi\right)+\phi L_{g_{2}} L_{g_{1}} \phi=\phi\left(L_{\left[g_{2}, g_{1}\right]} \phi+L_{g_{1}} L_{g_{2}} \phi\right)=0
\end{aligned}
$$

In the same way, we have

$$
\begin{aligned}
L_{g_{2}} L_{f}^{i} h & =0, \text { for } i=3, \cdots, n-2 \\
L_{g_{2}} L_{f}^{n-1} h & =\phi^{n-2} L_{a d_{g_{1}}^{n-2} g_{2}} \phi \neq 0
\end{aligned}
$$

Define new coordinate $z$ and new input $v$ respectively as

$$
\begin{aligned}
& z=\left[h, L_{f} h, L_{f}^{2} h, \cdots, L_{f}^{n-1} h\right]^{T} \\
& v=L_{f}^{n} h+u_{2} L_{g_{2}} L_{f}^{n-1} h
\end{aligned}
$$

The state space description of system (4) in the $z$ coordinates is then given by

$$
\left\{\begin{array}{ccc}
\dot{z}_{1} & =z_{2} \\
& \vdots \\
\dot{z}_{n-1} & = & z_{n} \\
\dot{z}_{n} & = & v
\end{array}\right.
$$

which is exactly the single-input Brunowsky canonical system.

The above analysis shows that, under state feedback control

$$
\left\{\begin{array}{l}
u_{1}=\phi(x) \\
u_{2}=\left(L_{g_{2}} L_{f}^{n-1} h(x)\right)^{-1}\left(v-L_{f}^{n} h(x)\right)
\end{array}\right.
$$

and coordinate transformation (5), system (3) is transformed into single-input controllable system (6). Because the input channel $u_{1}$ is pure state feedback, the overall input transformation $(7)$ is nonregular. $\diamond$ 
As a direct application of Lemma 1, we claim that the nonholonomic chained system

$$
\left\{\begin{aligned}
\dot{x}_{1} & =u_{1} \\
\dot{x}_{2} & =u_{2} \\
\dot{x}_{3} & =x_{2} u_{1} \\
& \vdots \\
\dot{x}_{n} & =x_{n-1} u_{1}
\end{aligned}\right.
$$

is nonregular feedback linearizable. Moreover, the linearizing output $h(x)$ and the real-valued function $\phi(x)$ in the proof of Lemma 1 could be explicitly constructed, say

$$
\begin{aligned}
h(x) & =x_{1} \\
\phi(x) & =\phi_{1}\left(x_{1}, x_{n}\right) \text { with } \frac{\partial \phi_{1}}{\partial x_{n}} \neq 0
\end{aligned}
$$

As the restriction imposed on $\phi(x)$ by (9) is not very restrictive, we can fully explore the freedom in controller design in practice. Different choices of $\phi(x)$ will result in different linearizing feedback transformations. In the next section, we will utilize this flexibility to develop different stabilization policies for nonholonomic chained systems.

\section{Stabilization of Wheeled Mobile Robots}

Consider the kinematic model of wheeled mobile robots that is transformed into chained form [5]:

$$
\left\{\begin{array}{l}
\dot{x}_{1}=u_{1} \\
\dot{x}_{2}=u_{2} \\
\dot{x}_{3}=x_{2} u_{1}
\end{array}\right.
$$

As examples, two stabilizing strategies in the framework of nonregular feedback linearization shall be proposed. One utilizes a discontinuous state transformation with smooth inverse, while the other uses a smooth state transformation with discontinuous inverse. Both control laws render the origin exponentially convergent. 


\subsection{Linearization with Discontinuous State Transformation}

Consider the nonsmooth function $\phi(x)=x_{3^{\frac{1}{3}}}$. From Lemma 1, we have

$$
u_{1}=x^{\frac{1}{3}}
$$

and system (10) is changed into a single-input nonlinear system.

Let $h(x)=x_{1}$. From (5), we have discontinuous state and input transformations

$$
\begin{array}{r}
\left\{\begin{array}{l}
z_{1}=x_{1} \\
z_{2}=x_{3}^{\frac{1}{3}} \\
z_{3}=\frac{1}{3} x_{2} x_{3}{ }^{-\frac{1}{3}}
\end{array}\right. \\
v=\frac{1}{3} x_{3}^{-\frac{1}{3}} u_{2}-\frac{1}{9} x_{2}^{2} x_{3}^{-1}
\end{array}
$$

which have discontinuous surface $S_{D}=\left\{z: z_{2}=0\right\}$.

The inverses of the state and input transformations are

$$
\begin{gathered}
\left\{\begin{array}{l}
x_{1}=z_{1} \\
x_{2}=3 z_{2} z_{3} \\
x_{3}=z_{2}^{3}
\end{array}\right. \\
\begin{cases}u_{1}=z_{2} \\
u_{2}=3 z_{2} v+3 z_{3}^{2}\end{cases}
\end{gathered}
$$

which are smooth.

The corresponding transformed linear system is of Brunovsky canonical form

$$
\left\{\begin{array}{l}
\dot{z}_{1}=z_{2} \\
\dot{z}_{2}=z_{3} \\
\dot{z}_{3}=v
\end{array}\right.
$$

For the simple linear system (16), controller design can be carried out easily. Let us assign them to be $-\lambda_{1},-\lambda_{2}$ and $-\lambda_{3}$ with $0<\lambda_{1}<\lambda_{2}<\lambda_{3}$. Accordingly, the controller is given by

$$
v=-\tau_{3} z_{1}-\tau_{2} z_{2}-\tau_{1} z_{3}
$$

where $\tau_{1}=\lambda_{1}+\lambda_{2}+\lambda_{3}, \tau_{2}=\lambda_{1} \lambda_{2}+\lambda_{1} \lambda_{3}+\lambda_{2} \lambda_{3}, \tau_{3}=\lambda_{1} \lambda_{2} \lambda_{3}$. 
Define set $D_{1}$ as

$D_{1}=\left\{x \in \Re^{3}:-\lambda_{1} \lambda_{2} x_{1} x_{3}^{\frac{1}{3}}-\left(\lambda_{3}+\frac{\lambda_{1} \lambda_{2}}{\lambda_{3}}\right) x_{3}{ }^{\frac{2}{3}}<\frac{1}{3} x_{2}<-\lambda_{2} \lambda_{3} x_{1} x_{3}{ }^{\frac{1}{3}}-\left(\lambda_{2}+\lambda_{3}\right) x_{3}{ }^{\frac{2}{3}}\right\}$

The closed-loop convergence is summarized in Theorem 1.

Theorem 1 If $x(0) \in D_{1}$, then the feedback control law

$$
\left\{\begin{array}{l}
u_{1}=x_{3}^{\frac{1}{3}} \\
u_{2}=-\tau_{1} x_{2}-3 \tau_{2} x_{3}{ }^{\frac{2}{3}}-3 \tau_{3} x_{1} x_{3}^{\frac{1}{3}}+\frac{1}{3} x_{2}^{2} x_{3}{ }^{-\frac{2}{3}}
\end{array}\right.
$$

derives the chained system (10) to the origin at an exponential rate with bounded input.

Proof. Note that the linearizing transformations (12) and (13) are well-defined if the trajectory of $z(t)$ do not cross the discontinuous surface $S_{D}$. Note also that $x(0) \in D_{1}$ if and only if $z(0) \in D_{0}$ with

$$
D_{0}=\left\{z: z_{2}\left(\lambda_{1} \lambda_{2} z_{1}+\frac{\lambda_{1} \lambda_{2}+\lambda_{3}^{2}}{\lambda_{3}} z_{2}+z_{3}\right)>0, z_{2}\left(\lambda_{2} \lambda_{3} z_{1}+\left(\lambda_{2}+\lambda_{3}\right) z_{2}+z_{3}\right)<0\right\}
$$

Consider the closed-loop system of (16) and (17). It is straightforward to calculate that

$$
z_{2} \stackrel{\text { def }}{=} a e^{-\lambda_{1} t}+b e^{-\lambda_{2} t}+c e^{-\lambda_{3} t}
$$

where

$$
\begin{aligned}
a & =\frac{-1}{\left(\lambda_{2}-\lambda_{1}\right)\left(\lambda_{3}-\lambda_{1}\right)}\left[\tau_{1} z_{1}(0)+\lambda_{1}\left(\lambda_{2}+\lambda_{3}\right) z_{2}(0)+\lambda_{1} z_{3}(0)\right] \\
b & =\frac{1}{\left(\lambda_{2}-\lambda_{1}\right)\left(\lambda_{3}-\lambda_{2}\right)}\left[\tau_{1} z_{1}(0)+\lambda_{2}\left(\lambda_{3}+\lambda_{1}\right) z_{2}(0)+\lambda_{2} z_{3}(0)\right] \\
c & =\frac{-1}{\left(\lambda_{3}-\lambda_{1}\right)\left(\lambda_{3}-\lambda_{2}\right)}\left[\tau_{1} z_{1}(0)+\lambda_{3}\left(\lambda_{1}+\lambda_{2}\right) z_{2}(0)+\lambda_{3} z_{3}(0)\right]
\end{aligned}
$$

To guarantee $z_{2}(t) z_{2}(0)>0$, the following conditions suffice

$$
z_{2}(0) a>0, z_{2}(0)(a+b)>0, z_{2}(0)(a+b+c)>0
$$

Routine calculation shows that this is equivalent to (19). Therefore, the linearizing transformations are well-defined. From (14), state $x(t)$ is bounded and converges to the origin exponentially. From (15), input $u(t)$ also exponentially converge to zero, and is bounded. 
Remark 1 Note that set $D_{1}$ is invariant under the feedback law (18). That is, every trajectory starting from $D_{1}$ will stay in $D_{1}$ for good. To see this, it suffices to show that set $D_{0}$ is an invariant set of the closed-loop system (16)-(17). Routine calculation gives that, for any $t \geq 0$, we have

$$
\begin{aligned}
& \lambda_{1} \lambda_{2} z_{1}(t)+\frac{\lambda_{1} \lambda_{2}+\lambda_{3}^{2}}{\lambda_{3}} z_{2}(t)+z_{3}(t)=\frac{\left(\lambda_{3}-\lambda_{1}\right)\left(\lambda_{3}-\lambda_{2}\right)}{\lambda_{3}}\left(a e^{-\lambda_{1} t}+b e^{-\lambda_{2} t}\right) \\
& \lambda_{2} \lambda_{3} z_{1}(t)+\left(\lambda_{2}+\lambda_{3}\right) z_{2}(t)+z_{3}(t)=\frac{-\left(\lambda_{2}-\lambda_{1}\right)\left(\lambda_{3}-\lambda_{1}\right)}{\lambda_{1}} a e^{-\lambda_{1} t}
\end{aligned}
$$

which, together with (21) and $z_{2}(0) z_{2}(t)>0$, yield

$$
\begin{aligned}
& z_{2}(t)\left(\lambda_{1} \lambda_{2} z_{1}(t)+\frac{\lambda_{1} \lambda_{2}+\lambda_{3}^{2}}{\lambda_{3}} z_{2}(t)+z_{3}(t)\right)>0 \\
& z_{2}(t)\left(\lambda_{2} \lambda_{3} z_{1}(t)+\left(\lambda_{2}+\lambda_{3}\right) z_{2}(t)+z_{3}(t)\right)<0
\end{aligned}
$$

which is exactly $z(t) \in D_{0}$ for all $t \geq 0$.

To steer the chained system (10) globally convergent, we only need to drive any initial configuration into set $D_{1}$ by an appropriate control input. For example, if $x_{2}(0) \neq 0$, one may first let $u_{2}=-k_{1} \operatorname{sgn}\left(x_{2}\right)$ to achieve $x_{2}=0$, then apply $u_{1}=-k_{2} \operatorname{sgn}\left(x_{3}\right)$ until $\lambda_{3} x_{1} x_{3}^{\frac{1}{3}}+x_{3}^{\frac{2}{3}}<0$, and finally make

$$
u_{2}=-k_{3} \operatorname{sgn}\left(x_{2}+\lambda_{3} x_{3}^{\frac{2}{3}}+\frac{3 \lambda_{2}\left(\lambda_{1}+\lambda_{3}\right)}{\lambda_{3}}\left(\lambda_{3}+x_{3}^{\frac{1}{3}}\right) x_{3}^{\frac{2}{3}}\right)
$$

which will steer the state trajectory into $D_{1}$.

Denote the respective control laws as

$$
\begin{aligned}
& u^{1}=\left[\begin{array}{c}
0 \\
-k_{1} \operatorname{sgn}\left(x_{2}\right) \\
-k_{2} \operatorname{sgn}\left(x_{3}\right) \\
0
\end{array}\right] \\
& u^{2}=\left[\begin{array}{c}
0 \\
-k_{3} \operatorname{sgn}\left(x_{2}+\lambda_{3} x_{3}^{\frac{2}{3}}+\frac{3 \lambda_{2}\left(\lambda_{1}+\lambda_{3}\right)}{\lambda_{3}}\left(\lambda_{3}+x_{3}^{\frac{1}{3}}\right) x_{3}^{\frac{2}{3}}\right) \\
x_{3}^{\frac{1}{3}}
\end{array}\right] \\
& u^{3}=\left[\begin{array}{c}
u^{4} \\
-\tau_{1} x_{2}-3 \lambda_{1} \lambda_{2} \lambda_{3} x_{1} x_{3}^{\frac{1}{3}}-3 \tau_{2} x_{3}{ }^{\frac{2}{3}}+\frac{1}{3} x_{2}^{2} x_{3}{ }^{-\frac{2}{3}}
\end{array}\right]
\end{aligned}
$$

Define the logic-based switching controller as

$$
u= \begin{cases}u^{4} & \text { if } x \in D_{1} \\ u^{3} & \text { if } x \notin D_{1} \text { and } \lambda_{3} x_{1} x_{3}^{\frac{1}{3}}+x_{3}^{\frac{2}{3}}<0 \\ u^{1} & \text { if } x \notin D_{1} \text { and } \lambda_{3} x_{1} x_{3}^{\frac{1}{3}}+x_{3}^{\frac{2}{3}} \geq 0 \text { and } x_{2} \neq 0 \\ u^{2} & \text { else }\end{cases}
$$


This controller steers system (10) globally convergent. In view of Remark 1, the total number of switching is less or equal to three.

Remark 2 For linear system (16), the feedback law (17) assigns distinct and realvalued poles. Other choices of pole location, though straightforward for linear system (16), may lead to more complicated situation in determining the invariant set $D_{0}$ and finding the finite switching strategy (23). In particular, if (a couple of) poles are complex-valued, the situation becomes too complicated to handle.

\subsection{Linearization with Smooth State Transformation}

As stated in Section 2, different choices for $\phi(x)$ satisfying (9) may result in different state transformations and the corresponding linearizing feedbacks. Due to the nonholonomic nature of system (10), if the state transformation is nonsingular, then its inverse must be discontinuous. Similarly, if the state transformation is smooth, then its inverse must be singular. Apart from the choice made in Subsection 3.1, smooth change of coordinates with singular inverse is also feasible.

Consider the smooth function $\phi(x)=x_{3}-\lambda_{1} x_{1}$ with $\lambda_{1}>0$. From Lemma 1, we have

$$
u_{1}=x_{3}-\lambda_{1} x_{1}
$$

and system (10) is transformed into a single-input nonlinear system.

Let $h(x)=x_{1}$. From (5), we have the smooth state and input transformations

$$
\begin{gathered}
\left\{\begin{array}{l}
z_{1}=x_{1} \\
z_{2}=x_{3}-\lambda_{1} x_{1} \\
z_{3}=\left(x_{2}-\lambda_{1}\right)\left(x_{3}-\lambda_{1} x_{1}\right)
\end{array}\right. \\
v=\left(x_{3}-\lambda_{1} x_{1}\right) u_{2}-\left(x_{2}-\lambda_{1}\right)^{2}\left(x_{3}-\lambda_{1} x_{1}\right)
\end{gathered}
$$

Under the smooth state transformation (25) and nonregular feedback control (24) and (26), the transformed system is of Brunovsky canonical form (16). 
The inverses of the state and input transformations are

$$
\begin{aligned}
& \left\{\begin{array}{l}
x_{1}=z_{1} \\
x_{2}=\lambda_{1}+\frac{z_{3}}{z_{2}} \\
x_{3}=z_{2}+\lambda_{1} z_{1}
\end{array}\right. \\
& \left\{\begin{array}{l}
u_{1}=z_{2} \\
u_{2}=\frac{v}{z_{2}}-\frac{z_{3}^{2}}{z_{2}^{2}}
\end{array}\right.
\end{aligned}
$$

which are discontinuous.

Thus, linear system (16) is stabilizing by linear feedback control (17).

It can be verified that $z(0) \in D_{0}$ iff $x(0) \in D_{2}$ with

$$
D_{2}=\left\{x \in \Re^{3}: \lambda_{2}+\frac{\lambda_{1} \lambda_{2} x_{1}}{x_{3}-\lambda_{1} x_{1}}<\lambda_{1}-\lambda_{3}-x_{2}<\frac{\lambda_{1} \lambda_{2}}{\lambda_{3}}+\frac{\lambda_{2} \lambda_{3} x_{1}}{x_{3}-\lambda_{1} x_{1}}\right\}
$$

Theorem 2 If $x(0) \in D_{2}$, then the feedback control law

$$
\left\{\begin{array}{l}
u_{1}=x_{3}-\lambda_{1} x_{1} \\
u_{2}=-\left(\lambda_{2}+\lambda_{3}-\lambda_{1}\right) x_{2}-x_{2}^{2}-\frac{\lambda_{1} \lambda_{3} x_{3}}{x_{3}-\lambda_{1} x_{1}}
\end{array}\right.
$$

steers chained system (10) to the origin at an exponential rate with bounded input.

Proof. From (27) and the fact that $z(t)$ is exponentially convergent, $x_{1}(t)$ and $x_{3}(t)$ converge to the origin exponentially. Now we prove that $x_{2}(t)$ converge to zero.

Simple calculation gives (see (20))

$$
\begin{aligned}
& z_{2}(t)=\frac{1}{\left(\lambda_{2}-\lambda_{1}\right)\left(\lambda_{3}-\lambda_{1}\right)\left(\lambda_{3}-\lambda_{2}\right)}\left(a e^{-\lambda_{1} t}+b e^{-\lambda_{2} t}+c e^{-\lambda_{3} t}\right) \\
& z_{3}(t)=-\frac{1}{\left(\lambda_{2}-\lambda_{1}\right)\left(\lambda_{3}-\lambda_{1}\right)\left(\lambda_{3}-\lambda_{2}\right)}\left(a \lambda_{1} e^{-\lambda_{1} t}+b \lambda_{2} e^{-\lambda_{2} t}+c \lambda_{3} e^{-\lambda_{3} t}\right)
\end{aligned}
$$

Note that the assumption $x(0) \in D_{2}$ implies that $a \neq 0$. Therefore,

$$
x_{2}(t)=\frac{\lambda_{1} z_{2}(t)+z_{3}(t)}{z_{2}(t)}=e^{-\left(\lambda_{2}-\lambda_{1}\right) t} \theta(t)
$$

where $\theta(t)=\frac{b\left(\lambda_{1}-\lambda_{2}\right) e^{-\left(\lambda_{2}-\lambda_{1}\right) t}+c\left(\lambda_{1}-\lambda_{3}\right) e^{-\left(\lambda_{3}-\lambda_{1}\right) t}}{a+b e^{-\left(\lambda_{2}-\lambda_{1}\right) t}+c e^{-\left(\lambda_{3}-\lambda_{1}\right) t}}$ which bounded by a linear function of time. As a consequence, $x_{2}(t)$ approaches to zero exponentially.

In the same way, we may prove that $u_{2}(t)$ is bounded and converges to zero with an exponential rate. 
If $x_{0} \notin D_{2}$, then it can be steered into $D_{2}$ in finite time by applying some closed-loop control. For example, we may first apply $u_{2}=-k_{1} \operatorname{sgn}\left(x_{2}-\lambda_{1}\right)$ to achieve $x_{2}\left(t_{1}\right)=\lambda_{1}$ at $t_{1} \geq \frac{\left|\lambda_{1}-x_{2}(0)\right|}{k_{1}}$, then apply $u_{1}=-k_{2} \operatorname{sgn}\left(x_{3}-\lambda_{1} x_{1}\right)$ until $x_{1}\left(t_{2}\right)+\frac{\lambda_{3}}{x_{3}\left(t_{2}\right)-\lambda_{1} x_{1}\left(t_{2}\right)}<0$ with $t_{2}>t_{1}+\frac{x_{1}(0)\left(x_{3}(0)-\lambda_{1} x_{1}(0)\right)}{k_{2}\left(x_{3}(0)-\lambda_{1} x_{1}(0)\right)}$, and finally, the control

$$
u_{2}=-k_{3} \operatorname{sgn}\left(x_{2}-\lambda_{1}+\lambda_{3}+\frac{\lambda_{2}\left(\lambda_{1}+\lambda_{3}\right)\left(\lambda_{3}+x_{1}\left(x_{3}-\lambda_{1} x_{1}\right)\right)}{2 \lambda_{3}\left(x_{3}-\lambda_{1} x_{1}\right)}\right)
$$

will bring the state trajectory into $D_{2}$.

Denote the respective control laws as

$$
\begin{aligned}
& u^{1}=\left[\begin{array}{c}
0 \\
-k_{1} \operatorname{sgn}\left(x_{2}-\lambda_{1}\right)
\end{array}\right] \\
& u^{2}=\left[\begin{array}{c}
-k_{2} \operatorname{sgn}\left(x_{3}-\lambda_{1} x_{1}\right) \\
0
\end{array}\right] \\
& u^{3}=\left[\begin{array}{c}
0 \\
-k_{3} \operatorname{sgn}\left(x_{2}-\lambda_{1}+\lambda_{3}+\frac{\lambda_{2}\left(\lambda_{1}+\lambda_{3}\right)\left(\lambda_{3}+x_{1}\left(x_{3}-\lambda_{1} x_{1}\right)\right)}{2 \lambda_{3}\left(x_{3}-\lambda_{1} x_{1}\right)}\right) \\
x_{3}-\lambda_{1} x_{1} \\
-\left(\lambda_{2}+\lambda_{3}-\lambda_{1}\right) x_{2}-x_{2}^{2}-\frac{\lambda_{1} \lambda_{3} x_{3}}{x_{3}-\lambda_{1} x_{1}}
\end{array}\right]
\end{aligned}
$$

Define logic-based switching controller

$$
u= \begin{cases}u^{4} & \text { if } x \in D_{2} \\ u^{3} & \text { if } x \notin D_{2} \text { and } x_{1}\left(x_{3}-\lambda_{1} x_{1}\right)^{2}+\lambda_{3}\left(x_{3}-\lambda_{1} x_{1}\right)<0 \\ u^{1} & \text { if } x \notin D_{2} \text { and } x_{1}\left(x_{3}-\lambda_{1} x_{1}\right)^{2}+\lambda_{3}\left(x_{3}-\lambda_{1} x_{1}\right) \geq 0 \text { and } x_{2} \neq \lambda_{1} \\ u^{2} & \text { else }\end{cases}
$$

This controller steers system (10) globally convergent. As $D_{0}$ being an invariant set for the transformed linear system (16)-(17), $D_{2}$ is an invariant set for the original system (10)-(29). Accordingly, the total number of switching is less or equal to three.

\section{Control of General Single Chained Systems}

In general, the discontinuous state transformation mechanism can be extended to the more general case of nonholonomic single chained form (8) though the smooth 
state transformation approach is hard to visualize for higher order systems. The design steps are outlined as follows.

Let the first control be

$$
u_{1}=\phi(x)= \begin{cases}x_{n}^{\frac{1}{n}} & \text { if } n \text { is odd } \\ x_{n}^{\frac{1}{n+1}} & \text { if } n \text { is even }\end{cases}
$$

Denote $f(x)$ and $g(x)$ by

$$
\begin{aligned}
f(x) & =\left[\phi(x), 0, x_{2} \phi(x), x_{3} \phi(x), \cdots, x_{n-1} \phi(x)\right]^{T} \\
g(x) & =[0,1,0,0, \cdots, 0]^{T}
\end{aligned}
$$

Define the real-valued function $h(x)=x_{1}$, and new coordinates $z$ and input $v$ as

$$
\begin{aligned}
& z=T(x)=\left[h, L_{f} h, L_{f}^{2} h, \cdots, L_{f}^{n-1} h\right]^{T} \\
& v=L_{f}^{n} h+u_{2} L_{g} L_{f}^{n-1} h
\end{aligned}
$$

The explicit expressions for $z$ and $v$ can easily be calculated, though they become more and more tedious as $n$ increasing. Note that $T(x)$ is discontinuous on the surface $\left\{x \in \Re^{n}: x_{n}=0\right\}$.

It may be verified that the inverse of $z=T(x)$, denoted by $x=T^{-1}(z)$, is a vector function with polynomial components. Therefore, exponential convergence of $z(t)$ implies exponential convergence of $x(t)=T^{-1}(z(t))$.

Consider the Brunovsky canonical system (6). It is standard that we may find a linear feedback control

$$
v=-K v=-\sum_{i=1}^{n} k_{i} z_{i}
$$

to exponentially stabilize this system. Let $W$ denote the subset of $\Re^{n}$ such that every trajectory of the closed-loop system started from $W$ will never cross the surface $\left\{z \in \Re^{n}, z_{2}=0\right\}$.

Define $D=\left\{x \in \Re^{n}: T(x) \in W\right\}$. For nonholonomic system (8), and the following control law

$$
\left\{\begin{array}{l}
u_{1}=\phi(x) \\
u_{2}=-\left(L_{g} L_{f}^{n-1} h\right)^{-1}\left(L_{f}^{n} h+K T(x)\right)
\end{array}\right.
$$


every trajectory of the closed-loop system started from $D$ will exponentially approach to the origin.

To achieve global convergence, before applying control law (31), a control strategy must be exploited to drive an arbitrary configuration outside $D$ into $D$ in finite time. This strategy may depend on the parameters $k_{i}, i=1, \cdots, n$ and could be determined if the $k_{i}^{\prime} s$ are given.

\section{Simulation Studies}

In this section, simulation studies are carried out to demonstrate the effectiveness and procedure of the proposed controllers for three dimensional chained system (10).

Firstly, consider the case when the initial states $x(0)=[-4,1,-2]^{T} \in D_{2}$. Accordingly, smooth controller (29) can be used. Figure ?? shows the convergence of the states, while Figure ?? demonstrates the boundedness of the control signals. As shown in Figure ??, the first control channel never crosses zero, as a consequence, $x_{2}(t)$ monotonically approaches zero.

For the case when the initial state is outside sets $D_{1}$ and $D_{2}$, appropriate control laws have to be derived to drive into the system into them in a finite time, then the pure state feedback control (18) or (29) can be applied accordingly. For simplicity, let us consider the initial state $x(0)=[-3,-4,1]^{T} \notin D_{1}$, as a consequence, switching logic (23) can be used. Figure ?? shows the converging trajectories of the states, while Figure ?? demonstrates the discontinuous switching and boundedness of the control signals. Due to the switching of the control signals, the state trajectories are not continuous as shown in Figure ??.

\section{Conclusion}

In this paper, control design strategies have been proposed to exponentially stabilize the nonholonomic chained systems in the framework of nonregular feedback linearization. Two discontinuous control laws based on logic-driven switching have 
been presented for three dimensional chained systems and simulation study has also been provided to show the effectiveness of the control schemes. In addition, the discontinuous state transformation approach was extended to general single chained systems.

\section{References}

[1] A. Astolfi, "Discontinuous control of nonholonomic systems," Systems Control Lett., vol. 27, pp. 37-45, 1996.

[2] A. Astolfi and W. Schaufelberger, "State and output stabilization of multiple chained systems with discontinuous control," Systems Control Lett., vol. 32, pp. 49-56, 1997.

[3] A. M. Bloch, N. H. McClamroch and M. Reyhanoglu, "Controllability and stabilizability properties of a nonholonomic control systems," in Proc. 29th IEEE CDC, Honolulu, Hawaii, 1990, pp.1312-1314.

[4] R. W. Brockett, "Asymptotic stability and feedback stabilization," in Differential Geometric Control Theory, R. W. Brockett, R. S. Millman and H. J. Sussman, Eds., Boston: Birkhauser, 1983, pp. 181-208.

[5] G. Compion, G. Bastin and B. d'Andrea-Novel, "Structure properties and classification of kinematic and dynamic models of wheeled mobile robots," IEEE Trans. Robotics and Automation, vol. 12, pp. 47-62, 1996.

[6] C. Canudas de Wit and O. J. Sordalen, "Exponential stabilization of mobile robots with nonholonomic constraints," IEEE Trans. Automat. Contr., vol. 37, pp. 1791-1797, 1992.

[7] L. Gurvits and Z. X. Li, "Smooth time-periodic feedback solutions for nonholonomic motion planning," in Progress in Nonholonomic Motion Planning, Z. X. Li and J. Canny, Eds., Norwell, MA: Kluwer, 1993, pp.53-108. 
[8] H. Khennouf and C. Canudas de Wit, "Quasi-continuous exponential stabilizers for nonholonomic systems," in 13th IFAC World Congress, San Francisco, 1996, pp. $49-54$.

[9] A. J. Krener, "On the equivalence of control systems and the linearization of nonlinear systems," SIAM J. Contr., vol. 11, pp. 670-676, 1973.

[10] R. M. Murray and S. S. Sastry, "Nonholonomic motion planning: steering using sinusoids," IEEE Trans. Automat. Contr., vol. 38, pp. 700-716, 1993.

[11] G. Oriolo and Y. Nakamura, "Control of mechanical systems with secondorder nonholonomic constraints: Underactuated manipulators," in Proc. of 30th $C D C, 1993$, pp. 306-308.

[12] O. J. Sordalen and O. Egeland, "Exponential stabilization of nonholonomic chained systems," IEEE Trans. Automat. Contr., vol. 40, pp. 35-49, 1995.

[13] Z. Sun and X. Xia, "On nonregular feedback linearization," Automatica, vol. 33, pp. 1339-1344, 1997. 